FERMILAB-TM-2537-APC

\title{
Some issues on $g-2$ proton beam preparation
}

\author{
K.Y. Ng
}

(February 17, 2012)

\begin{abstract}
In the preparation of proton beam for the $g-2$ experiment, one debunched Booster batch will be captured into four 2.5-MHz bunches in the Recycler Ring. The initial capture voltage is determined in order to limit leakage from the $2.5-\mathrm{MHz}$ buckets. The $2.5-\mathrm{MHz}$ rf voltage required to produce four bunches each of total width $10 \mathrm{~ns}$ is computed. In order to accomplish the required beam preparation in Recycler Ring, the maximum emittance growth and bunch length for the present experiments at the Main Injector are estimated.
\end{abstract}




\section{Introduction}

A booster batch of $8453-\mathrm{MHz}$ bunches is injected into the Main Injector. The matching $53-\mathrm{MHz}$ rf voltage is reduced adiabatically to zero, while the booster batch is debunched into a flat band of protons of energy spread $\pm \Delta E_{0}$ and total width $4 \times 397.64 \mathrm{~ns}$. This flat beam is then captured adiabatically into 4 bunches using $2.5-\mathrm{MHz}$ rf. The final area of each of the 4 bunches will be smaller if area of this flat beam is small. Thus it is important to keep $\Delta E_{0}$ as small as possible, and this is the reason why adiabatic debunching is required before the $2.5-\mathrm{MHz}$ rf capture. If the initial booster bunches are of area $0.1 \mathrm{eVs}$ each, one quarter of the flat debunched beam (21 booster bunches) will be of area $2.1 \mathrm{eVs}$. The ideal energy spread of the flat beam will be $\Delta E_{0}=2.64 \mathrm{MeV}$. Since the area of the booster bunches are usually slightly larger than $0.1 \mathrm{eVs}$, and the debunching cannot be exactly adiabatic, we will treat the energy spread of the debunched beam as $\Delta E_{0}=3.0 \mathrm{MeV}$ in the discussion below.

\section{Initial 2.5-MHz rf voltage}

During the adiabatic debunching and adiabatic capture, some particles will leak outside the injected booster batch time region, i.e., the $4 \times 397.64-n s$ range. One way to limit the leakage is to start off by setting up a small initial $2.5-\mathrm{MHz}$ rf voltage $V_{0}$. This voltage will drive the particles towards the buckets it creates instead of leaking out from the booster-batch region. The bucket height set up by this voltage is

$$
\widehat{\Delta E}=\sqrt{\frac{2 e V_{0} \beta^{2} E}{\pi h|\eta|}},
$$

and is depicted in the left plot of Fig. 1, where we have used particle energy $E=8.9383 \mathrm{GeV}$, MI slip factor $\eta=-8.9150 \times 10^{-3}$, and 2.5-MHz rf harmonic $h=28$. For example, with $\mathrm{rf}$ voltage $V_{0}=2 \mathrm{kV}$, some particles in the original booster bunches can migrate to the bucket height of $\pm 6.72 \mathrm{MeV}$, which is larger than the $\pm 3 \mathrm{MeV}$ resulting from adiabatic debunching without the presence of $2.5-\mathrm{MHz}$ rf voltage. If we assume the the adiabatic debunching is slow enough, beam particles will fill up the $2.4-\mathrm{MHz}$ bucket. The area occupied by $1 / 4$ of the booster batch will be just the area of the bucket or

$$
A_{\text {bucket }}=\frac{4 \widehat{\Delta E} t_{b}}{\pi},
$$



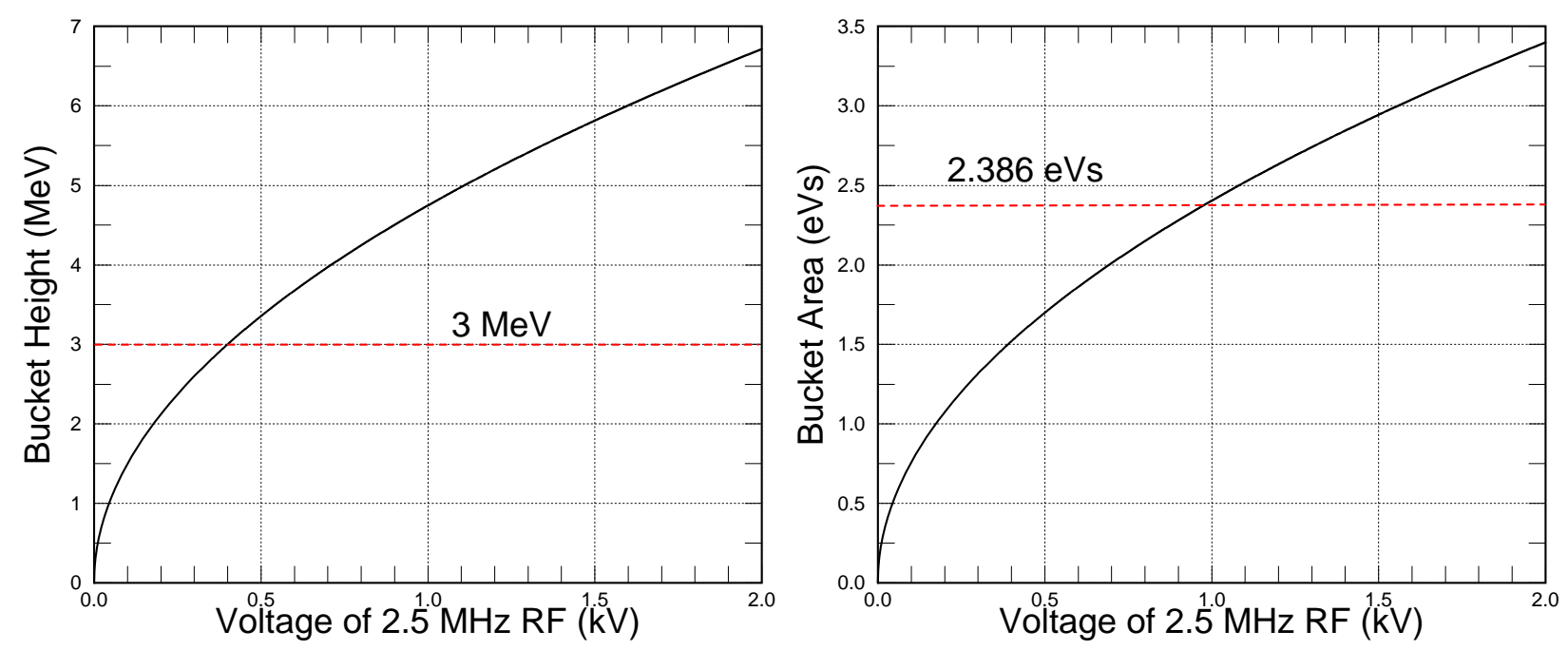

Figure 1: Left: Height of bucket set up by 2.5-MHz rf versus rf voltage. Right: Corresponding bucket area. The initial energy spread of $3 \mathrm{MeV}$ and beam area $2.386 \mathrm{eVs}$ are shown in red dashes.

which equals $3.40 \mathrm{eVs}$, where $t_{b}=397.64 \mathrm{~ns}$ is the bucket width or a quarter of the booster batch range (see right plot of Fig. 1). This is $42 \%$ larger than the area of the ideally adiabatic debunched beam. The captured $2.5-\mathrm{MHz}$ bunch area will increase at least by this amount even when the capture is perfectly adiabatic. We expect the captured bunch area will be much bigger than this because of the finite time devoted to the adiabatic capture. Experimental studies show that there is nearly no observable beam particle leakage at $V_{0}=$ $2 \mathrm{kV}$.

When the initial 25-MHz rf voltage is reduced to $V_{0}=1 \mathrm{kV}$, the bucket height it creates is $\widehat{\Delta E}=4.75 \mathrm{MeV}$ and the bucket area is $A_{\text {bucket }}=2.41 \mathrm{eVs}$, which is just slightly larger than the ideally adiabatic debunched area of $2.39 \mathrm{eVs}$. Experimental studies show a small amount of beam particle leakage. It is important to point out that not all beam particles will be captured inside the $2.5 \mathrm{MHz}$ rf bucket. Some will certainly drift outside the bucket resulting in a large beam emittance. As a result, it is important to keep the initial $2.5-\mathrm{MHz}$ rf voltage to below $V_{0}=1 \mathrm{kV}$, preferably $0.75 \mathrm{kV}$, for example.

To limit the amount of particles drifting outside the 2.5-MHz bucket, the input booster bunches should have as small an energy spread as possible. This implies that the final beam rotation before extraction at the booster should be avoided. Usually, when a longer bunch is rotated to a shorter bunch, there will be an increase in emittance due to first the nonlinearity of the rf wave, and second the space-charge force of the beam. Furthermore, a 
longer bunch length an smaller energy spread will shorten the debunching time duration in the Main Injector.

\section{$3 \quad 2.5-\mathrm{MHz}$ rf voltage required}

The maximum maintainable 2.5-MHz rf voltage in the Main Injector is $V=60 \mathrm{kV}$. A bunch with area $A=2.386 \mathrm{eVs}$ will have a half bunch length $\tau$ given by

$$
A=\sqrt{\frac{128 e V \beta^{2} E}{\pi h^{3} \omega_{0}^{2}|\eta|}}[E(m)-(1-m) K(m)] .
$$

where $m=\sin ^{2} \pi \tau / t_{b}$ while $K(m)$ and $E(m)$ are complete elliptical functions of the first and second kind.* The above gives $\tau=52.05 \mathrm{~ns}$, which will be even larger because the adiabatic capture can never be perfect. This implies that the $60 \mathrm{kV} \mathrm{rf}$ is far from capable of delivering a bunch of total width 100 ns.

In the future when the beam preparation is performed at the Recycler Ring, a new 2.5$\mathrm{MHz}$ rf system will be installed. Here we wish to compute the necessary rf voltage required. We wish eventually to produce a $2.5-\mathrm{MHz}$ bunch of half width $\tau=50 \mathrm{~ns}$ or less. The maximum allowable energy spread is $0.25 \%$, or $\Delta E=22.35 \mathrm{MeV}$, which is energy spread aperture of the Recycler Ring. The bunch area is

$$
A=\frac{4 t_{b} \Delta E}{\pi} \frac{[E(m)-(1-m) K(m)]}{\sqrt{m}}
$$

or $3.487 \mathrm{eVs}$. The rf voltage $V_{m}$ required to match such a bunch is given by Eq. (3.3), and we obtain $V_{m}=149.6 \mathrm{kV}$. If we start with a debunched beam of $\Delta E= \pm 3 \mathrm{MeV}$, or quarter debunched beam area $2.386 \mathrm{eVs}$, the allowable emittance growth is then $46 \%$. This gives us the guidance that in the present beam preparation experiment at the Main Injector, we must limit the emittance growth during adiabatic capture to $\leq 46 \%$. We learn that without emittance growth, the full bunch length obtainable from a $60-\mathrm{kV}$ rf is $104 \mathrm{~ns}$. This implies that we must at this moment produce a $2.5-\mathrm{MHz}$ bunch of width $\leq 104 \times \sqrt{1.46}=126 \mathrm{~ns}$. Looking back at the many study sessions, we obtained captured bunches of full width mostly about 140 to $150 \mathrm{~ns}$, which, of course, is not good enough. We did, however, accomplish capturing bunches of $\sim 120$ ns full width on the January 23 study, which is shown in Fig. 2

\footnotetext{
${ }^{*}$ When $m<<1,[E(m)-(1-m) K(m)] / \sqrt{m} \rightarrow \pi^{2} \tau /\left(4 t_{b}\right)$ and Eq. (3.3) reduces to $A=\pi \Delta E \tau$. Here $m=0.160$.
} 


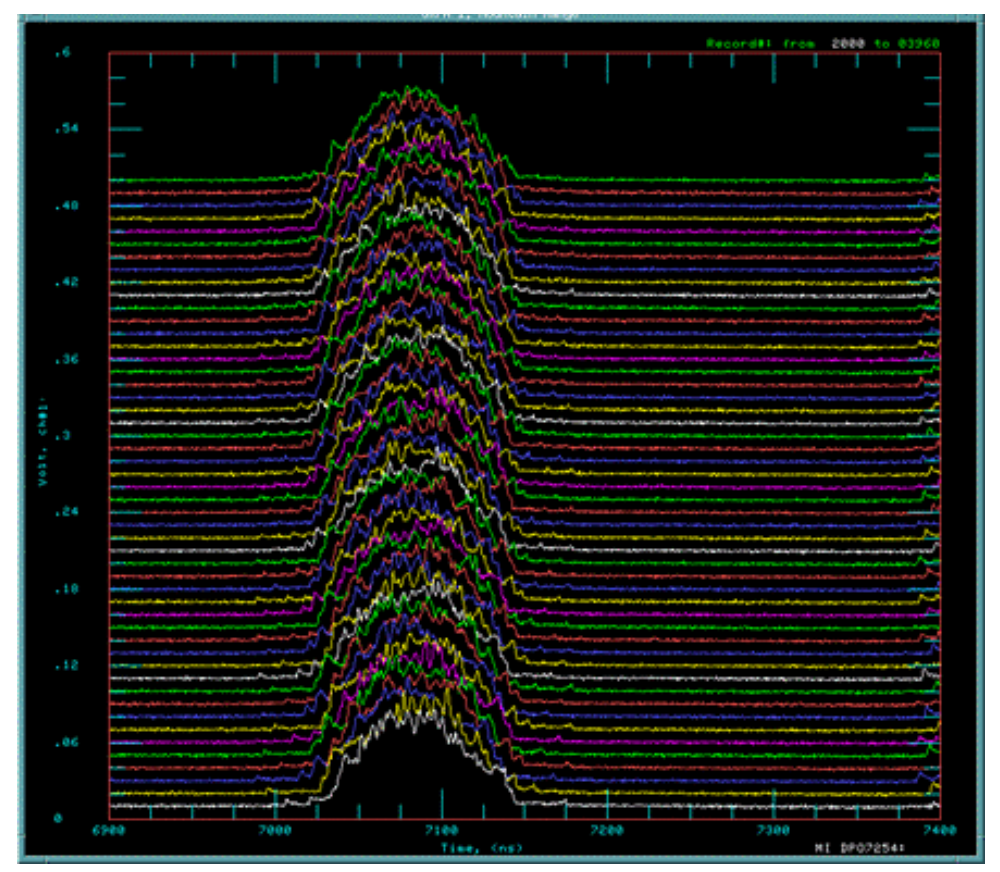

Figure 2: The first of four bunches captured by $2.5 \mathrm{MHz}$ rf from 1 to $60 \mathrm{kV}$ in $100 \mathrm{~ms}$. The captured full bunch length is $\approx 120$ ns. Each small division is $25 \mathrm{~ns}$. This study was made on January 23 , 2012.

\section{Conclusions}

We conclude that

1. The initial 2.5-MHz rf voltage must be less than $1 \mathrm{kV}$.

2. The final bunch rotation before extraction at the booster should be avoided.

3. Assuming an energy aperture of $0.25 \%$, the future $2.5-\mathrm{MHz}$ rf to be built for the Recycler Ring should have a voltage as high as $150 \mathrm{kV}$.

4. For our present studies at the Main Injector using 2.5-MHz rf voltage of $60 \mathrm{kV}$, we should try our best to limit emittance growth to less then $64 \%$, or the final full captured bunch width should be controlled to $\leq 126$ ns. 